\title{
VALIDITY AND RELIABILITY OF SHOULDER STRENGTH ASSESSMENT DURING SCAPTION, INTERNAL ROTATION AND EXTERNAL ROTATION USING AN ANCHORED, NON-MODIFIED SPHYGMOMANOMETER
}

original paper

(c) University School of Physical Education in Wroclaw

DOI: https://doi.org/10.5114/hm.2018.74064

\section{ALEXANDRE CARVALHO BARBOSA ${ }^{1}$, LEONARDO INTELANGELO ${ }^{2}$, DIEGO BORDACHAR $^{2}$, INGRID FERNANDES ${ }^{1}$, DENISE CARDOSO ${ }^{1}$, ILHA FERNANDES ${ }^{1}$, WYNGRID PORFIRIO ${ }^{1}$, DIOGO FELICIO ${ }^{1}$}

\author{
${ }^{1}$ Department of Physical Therapy, Musculoskeletal Research Group - NIME, Federal University of Juiz de Fora, Brazil \\ ${ }^{2}$ Department of Physical Therapy, Musculoskeletal Research Group - UIM, Instituto Universitario del Gran Rosario, \\ Argentina
}

\begin{abstract}
Purpose. To determine the validity, intra-rater reliability, and inter-rater reliability of an anchored sphygmomanometer for assessing shoulder strength during scaption, lateral rotation, and medial rotation. The hand-held dynamometer (HHD) was used as the standard measurement tool for this purpose.

Methods. Fifty subjects (23 years old \pm 3 years) were included in the validity study. Shoulder strength was assessed using an HHD and a sphygmomanometer, both anchored to a fixed ladder by an inextensible belt. Twenty-three subjects (25 years old \pm 3 years) were included in the reliability study. Two raters assessed strength, each taking two measurements one week apart, using the fixed sphygmomanometer.

Results. Validity results showed high to very high magnitude correlations, and no differences were found between the sphygmomanometer and the HHD measurements or among trials. Intra-class coefficient of correlation (ICC) showed high reliability between measurement tools and among trials. Intra-rater results showed very high ICC, very high correlation, low coefficient of variation (CV) with adequate standard error of measurement (SEM), and minimal detectable change (MDC). Inter-rater results showed moderate to high ICC, high to very high correlation, acceptable SEM and CV, but not adequate MDC. The anchored sphygmomanometer is a low-cost tool that provides objective measurements. The results obtained from the anchored sphygmomanometer were found to be similar to those obtained from an HHD, which has a valid predictive model. Conclusions. The sphygmomanometer is suitable for monitoring shoulder strength during scaption, internal rotation, and external rotation. The anchored sphygmomanometer enables coaches and physical therapists to establish the maximal voluntary isometric contraction and monitor exercise program outcomes at a low cost. However, caution is recommended when interpreting results between raters.
\end{abstract}

Key words: muscle, strength, dynamometer, sphygmomanometer, assessment

\section{Introduction}

Valid and reliable assessment tools enable physical therapists and coaches to accurately develop individualized exercise programs [1]. The strength assessment is especially important for providing data to inform a treatment diagnosis and accurate reporting of measurable outcomes for patients with neurological and musculoskeletal conditions. Additionally, muscle strength is correlated with a patient's functional capacity, and its accurate assessment provides objective data for measuring progress with intervention and informing discharge planning decisions [1,2].

Sufficient muscle strength is required around the shoulder girdle to perform activities of daily living by providing stabilization for elbow and wrist movement. This proximal stability allows refined movement at the hand and fingers, providing stable muscle syn-

Correspondence address: Alexandre Carvalho Barbosa, Department of Physical Therapy, Musculoskeletal Research Group - NIME, Federal University of Juiz de Fora, Brazil, e-mail: alexwbarbosa@hotmail.com

Received: July 25, 2017

Accepted for publication: December 12, 2017

Citation: Barbosa AC, Intelangelo L, Bordachar D, Fernandes I, Cardoso D, Fernandes I, Porfirio W, Felicio D. Validity and reliability of shoulder strength assessment during scaption, internal rotation and external rotation using an anchored, non-modified sphygmomanometer. Hum Mov. 2018;19(2):90-98; doi: https://doi.org/10.5114/hm.2018.74064. 
ergy that is essential to functional upper limb movement [3]. Shoulder girdle muscle weakness has been associated with supraspinatus tear and shoulder impingement syndrome [4] as well as anterior shoulder instability and superior labral tear from anterior to posterior [5]. Weak internal rotators are associated with humeral retroversion, posterior-inferior capsular contracture, and posterior rotator cuff tightness [6]. Also, the ratio of strength between the external and internal rotators affects the balance of the glenohumeral joint [7]. Muscle strength and length imbalances between these opposing muscle groups increase the risk for shoulder injuries. [8]. Scaption is also a functional movement used during daily activities and requires deltoid, supraspinatus, and serratus anterior muscle activation [9]. Scaption, internal rotation, and external rotation elicit high levels of muscle activity in healthy individuals and are thought to play a critical role in stabilizing the humeral head within the glenoid cavity [10]. The muscles activated during these shoulder movements are assessed to determine the condition of the rotator cuff and inform the therapist on where to focus the patient's strengthening program. An objective, low-cost assessment to detect muscle weakness could help the physical therapist during the rehabilitation process and prevent future impairments.

Muscle strength may be assessed using subjective or objective methods [11]. The manual muscle test is a subjective test most frequently used in clinical practice because it is easy to perform and has no cost. However, its reliability is low [12], and it does not provide objective parameters for exercise prescription or treatment outcomes measurement. The isokinetic dynamometer is the gold standard for muscle strength assessment, but it is extremely expensive [1]. The hand held dynamometer (HHD) is a portable option with validity and reliability comparable to isokinetic dynamometry when assessing isometric muscle strength in several joint movements $[1,13,14]$. It is important to note the equipment's anchoring method when the strength test is performed using an HHD in order to maintain the validity and reliability [15]. A recent study achieved higher validity and reliability by using an inextensible band to keep the HHD stable while assessing knee extension strength compared to the non-fixed HHD method [14] suggesting that this adaptation could be a better way to assess muscle strength compared to non-fixed equipment [16]. Despite its usefulness and lower cost compared to an isokinetic dynamometer, the HHD is still a relatively expensive tool.

A possible alternative to the HHD is the aneroid sphygmomanometer; a well-known and low-cost tool that is commonly acquired to assess blood pressure. Studies have shown moderate reliability of the sphygmomanometer, when compared to an HHD, to assess hand grip strength in Parkinson's disease [17], upper limb muscles after stroke [2] and isometric strength of hip muscles [18]. Some of those studies also showed adaptations on the sphygmomanometer, such as removing the inflatable cuff, to assess isometric muscle strength [17, 19, 20]. Nevertheless, it is important to perform strength tests without adaptations in the equipment to ensure its usefulness in daily assessments. To reinforce the tool's consistency, both adapted (the sphygmomanometer without the inflatable cuff) and non-adapted (manufacturer's original sphygmomanometer) methods showed significant and adequate correlations with the HHD, requiring only one repetition after familiarization, to properly assess the muscle strength in healthy individuals [20].

Both hand-held dynamometry and the sphygmomanometer test (ST) have been shown to be preferable alternatives over manual muscle tests [1, 21]. The HHD has been shown to detect weakness in shoulder external and internal rotator muscles deemed normal by manual muscle testing [22], but previous studies have only investigated the correlation between the ST and the HHD for upper limbs in patients after stroke [2, 23]. The present study aims to present novel insights about the use of the sphygmomanometer compared to the HHD. These aspects, previously unstudied, include anchoring the equipment to improve stabilization thereby ensuring values closer to those obtained using an anchored HHD. The study also aims to address issues not usually noted by other authors, such as attention to air displacement prevention and the recalibration procedure. To our knowledge, no studies are available regarding the validity of the non-adapted sphygmomanometer compared to HHD for shoulder lateral rotation, medial rotation and arm elevation in the scapular plane (scaption).

Therefore, the present study aims to assess validity, intra-rater reliability and inter-rater reliability of an anchored ST during shoulder scaption, lateral rotation and medial rotation using a hand-held dynamometer (HHD) as the standard tool of measurement.

\section{Material and methods}

\section{Experimental approach to the problem}

This study assessed the concurrent instrumental validation between the ST and the HHD. A second, parallel study assessed intra-rater and inter-rater re- 
A.C. Barbosa et al., Validity reliability sphygmomanometer shoulder

liability of the ST. The selected standard tool, the HHD, is suitable to assess the sphygmomanometer validity. The HHD showed minimal differences compared to isokinetic testing (gold standard) for muscle strength measurement $[1,24]$. Healthy young adults were tested twice, 1-week apart, by two raters. The relative reliability was assessed by intra-class coefficient of correlation (ICC) and Pearson's coefficient, exploring the agreement between raters and between measurements (intra-rater reliability). The absolute reliability provides the extent of measurement error by coefficient of variation $(\mathrm{CV})$, standard error of measurement (SEM) and minimal detectable change (MDC).

\section{Participants}

Two sample selections for each study were carried out by public call in the city of Governador Valadares Minas Gerais - Brazil. Table 1 summarizes the participants' characteristics for both studies. Inclusion criteria were 1) aged 30 years or less, 2) physically independent, and 3) scored higher than 21 on the MiniMental State Examination. Exclusion criteria were 1) self-reported shoulder pathologies, 2) cervical traumato-orthopedic injuries and/or 3) localized pain in the assessed region. Also, participants were excluded if unable to stabilize the continuous force applied over the sphygmomanometer. The usual procedure consisted of no consideration for the first impact over the sphygmomanometer, as it was usually higher than the average readings. Two participants were excluded due to inability to maintain continuous impacts over the sphygmomanometer during all trials. A post-hoc sample power analysis was carried with an effect size of 0.87 (derived from the weakest correlation analysis with $r=0.77$ and sample size of 20), the alpha was set at 0.05 , and the sample power was calculated using the G-Power software (Version 3.1.5, Franz Faul, Universitat Kiel, Germany). The power twotailed analysis returned an actual power of 0.99 .

Table 1. Participants' characteristics

\begin{tabular}{lcc}
\hline Characteristics & Validity & $\begin{array}{c}\text { Intra/inter-rater } \\
\text { reliability }\end{array}$ \\
\hline Sample size & $n=50$ & $n=23$ \\
Age (years-old) & $24(3)$ & $25(3)$ \\
Height $(\mathrm{cm})$ & $170(9)$ & $171(9)$ \\
Weight $(\mathrm{Kg})$ & $69(13)$ & $66(14)$ \\
Male/Female & $24 / 26$ & $12 / 11$ \\
\hline
\end{tabular}

mean (standard deviation)

\section{Materials}

The maximum voluntary isometric contraction (MVIC) was assessed through an HHD (Nicholas Manual muscle test, Co, Lafayette IN), considered the standard tool [20, 23, 25]. The tested tool was a new aneroid sphygmomanometer with a measuring range from 0 to $300 \mathrm{mmHg}$, division scale of $2 \mathrm{mmHg}$, cuff size $7 \times 17 x 11 \mathrm{~cm}$, clamp in nylon non-allergic fabric, with pin locking, properly calibrated and checked by Brazilian National Institute of Metrology, Quality and Technology (INMETRO). Both instruments were positioned inside an inextensible belt which was fixed to a fixed ladder. This decision was based on a previous study that demonstrated greater reliability of MVIC measurements of the shoulder with an HHD when the tool was fixed with an inextensible belt [13].

\section{Procedures}

Tests were performed using the sphygmomanometer and the HHD, respectively, with 15-minute intervals between each test. Two randomly assigned examiners (https://www.randomizer.org/) positioned the equipment inside the fixed belt, a third examiner read the values and a fourth one recorded and stored the results. For each movement, subjects were asked to push as hard as possible against the assessment device. The tests consisted of 3 trials of MVIC for 6 seconds each, with one minute of rest between trials. All tests were performed on the volunteers' dominant upper limbs. The dominant upper limb was determined as the limb most frequently used to write. During the ST an assistant read and recorded the results, and another assistant controlled the chronometer. Calibration procedures were performed before ST data collection using $5 \mathrm{~kg}$ weights to verify that the equipment provided consistent measurements throughout the study [20]. The calibration procedure was performed for each volunteer as follows: (1) the sphygmomanometer was inflated $(100 \mathrm{mmHg})$; (2) the pressure was reduced to $40 \mathrm{mmHg}$, and the valve was closed to prevent leakage, providing a measurement range of 20-300 mmHg. The examiner ensured the pre-inflation of the equipment before each subject's test. To assess the intra-rater reliability, one examiner performed the ST on two different occasions with 1-week between each test. Two examiners performed the ST independently on each participant to assess inter-rater reliability.

All tests were performed on participants in the standing position. For shoulder scaption, subjects placed 


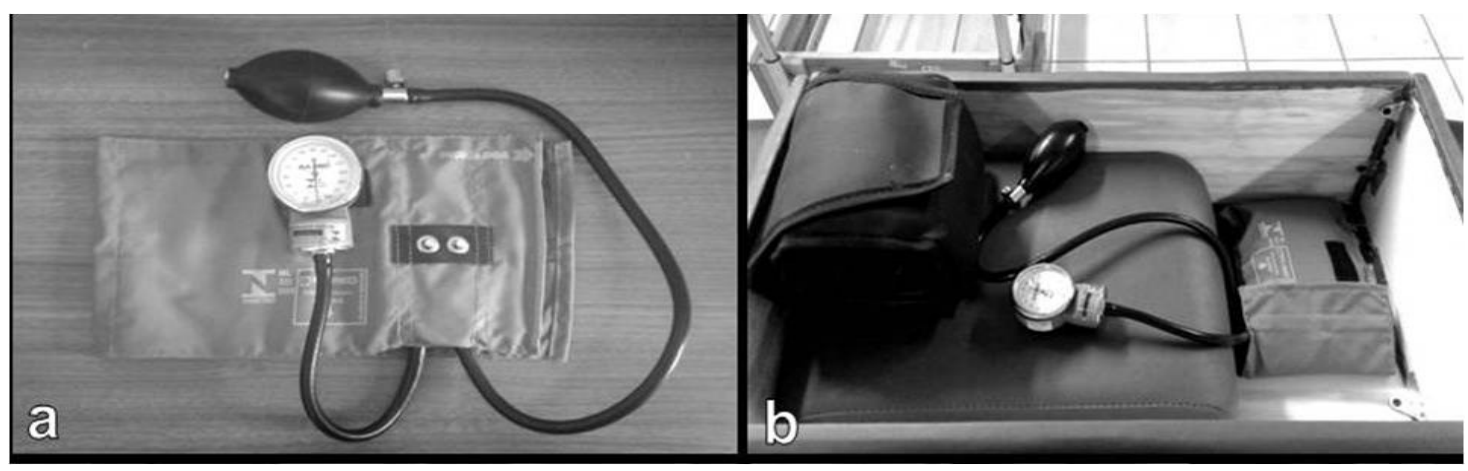

Figure 1. Aneroid sphygmomanometer (a), calibration apparatus (b)

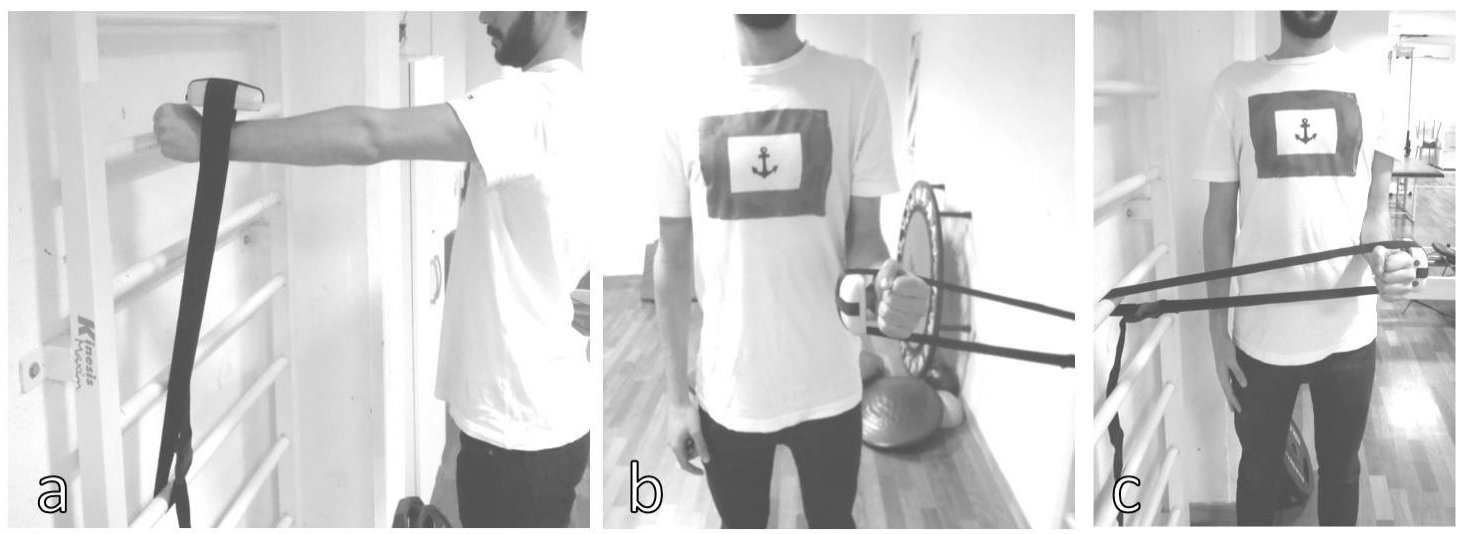

Figure 2. Hand Held Dynamometer test - scaption (a), internal rotation (b), external rotation (c)
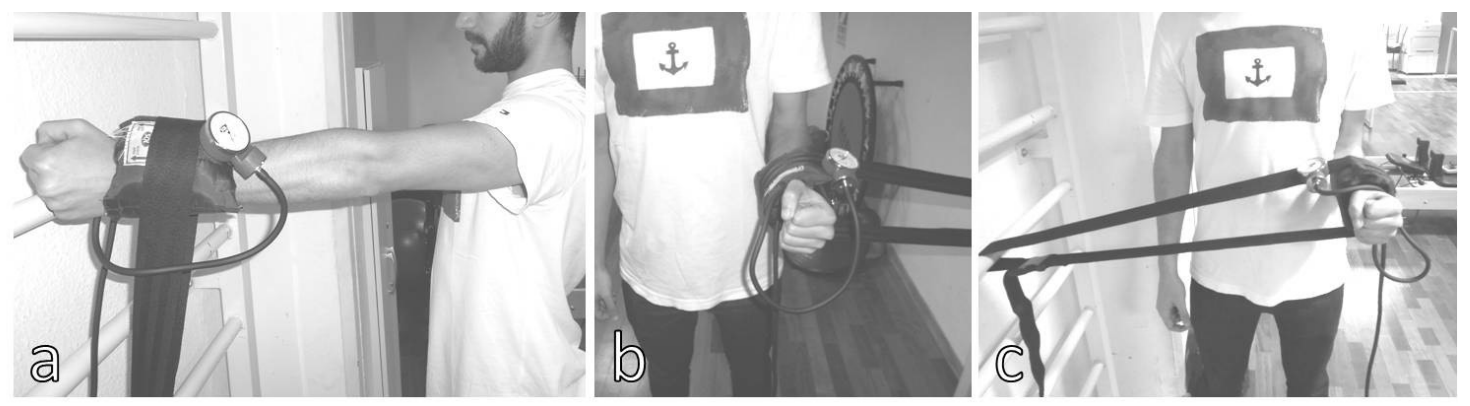

Figure 3. Sphygmomanometer test - scaption (a), internal rotation (b), external rotation (c)

their arms at 90 degrees of glenohumeral elevation in the "full can" position (full can position refers to the arm at $90^{\circ}$ of elevation in the scapular plane with glenohumeral external rotation and thumb up). The sphygmomanometer and the HHD were placed right above the distal forearm (at the wrist level). For shoulder internal and external rotation the arm was placed against the body with elbow flexed at 90 degrees. Both the sphygnamometer and the HHD were placed on the distal forearm (at the wrist level) internally and externally to respectively assess internal and external rotation. All tests were performed with the tools fixed to a belt which was anchored to a fixed ladder (Figure 1, 2 and 3).

The test was explained to each volunteer, and stand- ardized auditory stimulus was given to start the test by loudly repeating "go" during all tests. The first trial was used to familiarize the subject to the procedure, and the obtained mean values from the last 2 trials were used for statistical analysis.

\section{Statistical analysis}

The normality was assessed by the Shapiro-Wilk test. Descriptive data were presented by means and standard deviation. To analyze the concurrent validity, the linear regression analysis and the coefficient of determination were performed, and prediction equations of values in kilogram-force (Kgf) were established from data obtained through the sphygmomanom- 


\section{HUMAN MOVEMENT}

A.C. Barbosa et al., Validity reliability sphygmomanometer shoulder

eter in $\mathrm{mmHg}$. Values in $\mathrm{mmHg}$ were then converted to Kgf (sphygmomanometer's transformed values - tST). After conversion, the paired t-test was performed to assess differences between tST and HHD results. The correlation was calculated using the Pearson's coefficient. ANOVA 1-way and ICC were used to respectively assess differences and reproducibility among trials. The reliability was assessed using the ICC and the respective 95\% confidence interval (CI). To analyze the intra- and inter-rater agreement, the $\mathrm{CV}$, the Pearson's coefficient, the SEM and the MDC were used. The SEM reflects the instrument error and was calculated by dividing the standard deviation (SD) of the mean difference by the square root of 2 (SD of the differences $/ \sqrt{ } 2)$. The MDC can be interpreted as real change and was calculated using the formula MDC = $1.96 \times \sqrt{ } 2 \times$ SEM. The magnitude of the ICC and Pearson's coefficient of correlation were classified as follows: very low $(\leq 0.25)$, low $(0.26-0.49)$, moderate $(0.50$ 0.69), high (0.70-0.89) and very high (0.90-1.00) [23]. Significance level was established at $\mathrm{p}<0.05$. BioEstat 5.0 software was used for all statistical analysis.

\section{Ethical approval}

The research related to human use has been complied with all the relevant national regulations and institutional policies, has followed the tenets of the Declaration of Helsinki, and has been approved by the authors' institutional review board or an equivalent committee.

The local ethics committee approved this study (CAAE 63883116.1.0000.5147).

\section{Informed consent}

All participants were informed about all procedures and signed an informed consent form prior to participation.

\section{Results}

Validity

None of the subjects reported any localized muscle pain or fatigue. No differences and high ICC among trials were observed (scaption, $\mathrm{p}=0.62 / \mathrm{ICC}=0.75$; internal rotation $\mathrm{p}=0.70 / \mathrm{ICC}=0.85$; external rotation, $\mathrm{p}=0.58 / \mathrm{ICC}=0.85$ ). Table 2 shows ST and HHD descriptive results for each test (scaption, internal and external rotation) and the transformed data (tST) using the prediction equations obtained from the linear regression calculations. The coefficient of determination showed the equations as good predictors of values in $\mathrm{Kgf}$ from obtained values in $\mathrm{mmHg}$. In systematic bias analysis by paired t-tests, no differences were noted when comparing tST and those obtained from HHD (scaption, $p=0.99$; internal rotation, $p=0.98$; and external rotation, $p=0.98$ ).

Significant and positive high correlations were noted (Table 3) between the ST and the HHD for all movements. Very high reliability (ICC) between transformed values and HHD results are also shown in Table 3.

\section{Reliability}

Pearson's coefficient and ICC values denoted very high intra-reliability, with range above 0.90 . For ab-

Table 2. Descriptive data (mean and standard deviation) of scaption, internal and external rotation, regression equation and coefficient of determination

\begin{tabular}{lcrrrr}
\cline { 2 - 4 } & ST [mmHg] & tST [Kgf] & HHD [Kgf] & regression equation & $\mathrm{r}^{2}$ \\
\hline Scaption & $120(33)$ & $3.6(1.37)$ & $3.6(1.48)$ & $\mathrm{y}=0.0409 \mathrm{x}-1.2617$ & 0.85 \\
Internal Rotation & $134(39)$ & $5(1.65)$ & $5(2.02)$ & $\mathrm{y}=0.0427 \mathrm{x}-0.6777$ & 0.67 \\
External Rotation & $108(31)$ & $3.8(1.13)$ & $3.8(1.34)$ & $\mathrm{y}=0.0367 \mathrm{x}-0.1373$ & 0.71 \\
\hline
\end{tabular}

ST - sphygmomanometer test, tST - transformed sphygmomanometer, HHD - hand-held dynamometer; $\mathrm{r}^{2}-$ coefficient of determination

Table 3. Correlation and reliability between hand-held dynamometer and transformed sphygmomanometer data from scaption, internal and external rotation of the shoulder

\begin{tabular}{llll} 
& \multicolumn{1}{c}{$\mathrm{r}$} & $95 \%-\mathrm{CI}$ & ICC \\
\cline { 2 - 4 } Scaption & $0.92^{*}$ (very high) & $0.87-0.96$ & 0.89 (high) \\
Internal Rotation & $0.82^{*}$ (high) & $0.70-0.89$ & 0.78 (high) \\
External Rotation & $0.84^{*}$ (high) & $0.73-0.91$ & 0.81 (high) \\
\hline
\end{tabular}

r - Pearson's coefficient of correlation, 95\%-CI - confidence interval, ICC - intra-class correlation coefficient 
Table 4. Intra-rater reliability

\begin{tabular}{lccccccc}
\cline { 2 - 7 } & ICC & $95 \%-C I$ & $\mathrm{r}$ & $95 \%-\mathrm{CI}$ & $\mathrm{CV}(\%)$ & SEM & MDC \\
\hline Scaption & 0.95 & $0.88-0.98$ & 0.96 & $0.90-0.98$ & 5 & 3 & 8 \\
Internal Rotation & 0.92 & $0.82-0.97$ & 0.92 & $0.81-0.96$ & 5 & 4 & 12 \\
External Rotation & 0.96 & $0.90-0.98$ & 0.97 & $0.93-0.99$ & 3 & 2 & 6 \\
\hline
\end{tabular}

r - Pearson's coefficient of correlation, 95\%-CI - confidence interval, CV - coefficient of variation, SEM - standard error of measurement, $\mathrm{MDC}=$ minimal detectable change

Table 5. Inter-rater reliability

\begin{tabular}{lccccccc}
\cline { 2 - 7 } & ICC & $95 \%-C I$ & $\mathrm{r}$ & $95 \%-C I$ & CV(\%) & SEM & MDC \\
\hline Scaption & 0.83 & $0.64-0.92$ & 0.92 & $0.82-0.97$ & 9 & 6 & 15 \\
Internal Rotation & 0.67 & $0.35-0.84$ & 0.77 & $0.53-0.90$ & 12 & 9 & 26 \\
External Rotation & 0.87 & $0.71-0.94$ & 0.92 & $0.83-0.97$ & 8 & 5 & 14 \\
\hline
\end{tabular}

$\mathrm{r}$ - Pearson's coefficient of correlation, 95\%-CI - confidence interval, CV - coefficient of variation, SEM - standard error of measurement, MDC - minimal detectable change

solute reliability, the $\mathrm{CV}$ variation was above $10 \%$, the SEM of each movement ranged from 2 to $4 \mathrm{mmHg}$. The lowest MDC was 6, and the highest was $12 \mathrm{mmHg}$. All results denoted satisfactory intra-rater reliability (Table 4).

The inter-rater reliability showed relative reliability ICC values ranging from moderate - for internal rotation - to high - for scaption and external rotation - with Pearson's coefficient showing high to very high results. The absolute reliability showed internal rotation's CV above $10 \%$, with scaption and external rotation close to the limit. SEM ranged from 5 to $9 \mathrm{mmHg}$, and the MDC range was $14-26 \mathrm{mmHg}$. The absolute inter-rater reliability results suggest a systematic bias between the 2 raters.

\section{Discussion}

The results suggest that the fixed, non-adapted ST is a valid and intra-rater reliable alternative to measure muscle strength compared to the HHD technique during scaption, internal rotation and external rotation of the shoulder in healthy adults. Souza et al. [20] assessed the non-adapted sphygmomanometer as a tool able to provide valid muscle strength measurements for elbow flexion, knee extension and trunk flexion. However, the same authors reported difficulties with stabilizing the equipment during the MVIC for knee extension. This type of limitation is often reported in the literature using the HHD, with higher reliability achieved only when the rater is stronger than the subject [26, 27]. Studies using the HHD showed that the proficiency of the raters affects the results, and anchoring the HHD could avoid rater interference [15, 28, 29]. Both enhanced reliability and validity - as compared to the isokinetic dynamometer - were noted when the HHD was fixed compared to non-fixed trials [14]. The present study did not assess the same muscles, but the use of an inelastic belt provided stability and consistent results during ST compared to HHD tests. It is assumed that, when the belt was folded around the sphygmomanometer, its borders helped to secure the equipment, decreasing the point of contact against the limb's force compared to holding the equipment with hands. A uniform pressure was obtained when the belt was correctly folded around the center of the sphygmomanometer, leading to increased test stability, and avoiding air displacement under the belt. Thus, the anchoring procedure avoided rater interference as a potential source of bias.

Previous studies reported stability across ST results with no recalibration other than that recommended by the manufacturer [11, 20]. The current study checked the air content after each participant's tests and found air loss after every three measurements. As the calibration procedure is essential for valid and reliable values, our results suggest that new calibration for each patient is necessary to avoid wrong measurements. The adopted pre-inflation value (40 $\mathrm{mmHg}$ ) also diverges from other studies. The most used value is $20 \mathrm{mmHg}$, but another study also suggested different pre-inflation values [30]. Tests performed prior to data collection also showed better results with $40 \mathrm{mmHg}$. The non-adapted sphygmomanometer has a larger area of contact with the limb, providing lower pressure values when an external force is applied [20]. 
A.C. Barbosa et al., Validity reliability sphygmomanometer shoulder

This issue became more evident using the anchored inelastic belt due to the enhanced stabilizing force applied through the surrounding area of contact and not only against the limb's counterforce. Higher pressure value was then necessary for proper calibration.

Reliability studies are essential to assess the variability of an instrument to avoid misinterpretation of variables before and after interventions [17]. A previous study showed excellent test-retest reliability of the ST for shoulder internal and external rotator strength in healthy subjects (ICC > 0.90) [21]. However, a literature search found no studies that assessed the reliability of the ST for scaption. Six scapular muscles are fired to increase the stability of the humeral head during scaption [31]. In this movement, the arm is elevated with full elbow extension and glenohumeral external rotation, i.e., the "full can" position, as opposed to the "empty can" position in which the thumb points to the ground (shoulder in internal rotation). It has been shown that elevating the arm in shoulder external rotation is associated with less middle deltoid muscle activity and higher supraspinatus muscle activity, reducing the superior-anterior subluxation of the humeral head. Also, external rotation moves the greater tuberosity away from the undersurface of the acromion, increasing the width of the subacromial space. These actions decrease the risk of impingement [32]. Intra-rater reliability confirmed the consistency when the same evaluator performed the procedure on different days of data collection. The inter-rater agreement, when assessed using the SEM and MDC, showed larger MDC values than the intra-rater results. The assessments performed at different times in the same individual would have variations ranging from 5 to 9 mmHg (SEM), depending on the movement, and related to measurement errors as opposed to changes in the strength status. Compared to intra-rater variations ( 2 to $4 \mathrm{mmHg}$ ) and considering the $2-\mathrm{mmHg}$ scale, the range was larger but not too discrepant. Also, the CV showed acceptable levels of variation, ranging from 5 to $9 \%$ among the results, not very different when compared to intra-rater trials (3 to 5\%). However, the MDC showed ranges from 14 to $26 \mathrm{mmHg}$. The results indicate that changes greater than 14 to $26 \mathrm{mmHg}$, depending on the movement, has a probability of $5 \%$ of occurrence due to a random error or a random variation, very different when comparing the intra-rater results, with a range from 6 to $12 \mathrm{mmHg}$. The absolute reliability demonstrated that when assessing the shoulder movements by different raters, there is great variation with low agreement and important measurement error. Results should be interpreted with caution if the goal is to compare different rater assessments.
The present study used means from two last trials for statistical analysis - as more repetitions could reduce errors [33] - and longer resting time (1 minute among trials) compared to other studies (15-20 seconds on average) [19, 20, 23] to allow recovery after MVIC. No differences between trials were observed. Other studies also noted no difference among measurements considering multiple trials, recommending one trial after familiarization to avoid fatigue and/or pain [20, 23]. Nevertheless, participants did not report fatigue or discomfort after the experiment (familiarization +2 trials). We recommend two or more trials due to subject's adaptation to the task. Two participants were excluded due to their lack of control during all trials, suggesting the need for more familiarization. Some subjects also tried to compensate by elevating the scapula or bending the trunk to increase the generated force, mainly during scaption. The examiner was aware about compensations and instructed all participants to correct their postures. Although the current shoulder and body positions for strength assessment have been studied and standardized, the assessment of shoulder strength performed with the sphygmomanometer is not free from common issues described during other strength assessments. The equipment stabilization with a belt, the required attention to prevent air displacement and the frequent recalibration are potential disadvantages of the ST. However, the method provided a valid, intra-rater reliable, low cost and portable method for shoulder strength assessment. These advantages should be considered to include the ST in clinical practice to replace manual muscle testing.

A limitation of the present study includes that the results were read and recorded by an independent assistant to ensure the internal validity of the study by blinding the principal examiner, although Martins et al. [19] adopted the same procedure. The sample also included only young, healthy participants. Further studies must include samples with different ages and pathologies to confirm the outcome. Additional limitations include not testing the equipment against the gold standard isokinetic dynamometer and testing only on upper extremities.

In conclusion, we recommend measuring shoulder strength using the sphygmomanometer which provides objective, low-cost and similar strength measurement results to those obtained from the HHD. The results from the non-adapted sphygmomanometer presented significant positive correlation and similar magnitude to those obtained by using an HHD in young, healthy subjects with valid predictive models. These models showed similar results to those obtained 
using the HHD. Inter-rater comparisons should be interpreted with caution.

The sphygmomanometer is a convenient, portable, valid, and intra-rater reliable tool for measuring muscle strength during scaption, internal rotation and external rotation of the shoulder. This information may assist coaches and physical therapists with exercise program planning and outcomes assessments. The strength protocol can be monitored at a low cost to establish the MVIC and its prescribed percentage to enhance strength, endurance and/or motor control. The present study used an inextensible belt anchored to a fixed ladder which provided stabilization of the sphygmomanometer during the strength tests. The stabilization of test instruments seemed to play an important role in providing reliable measurements. Also, the present study clarifies necessary actions to take when performing the ST including subject's familiarization, examiner's attention to prevent air and equipment displacement, and recalibration between tests.

\section{Disclosure statement}

No author has any financial interest or received any financial benefit from this research.

\section{Conflict of interest}

The authors state no conflict of interest.

\section{References}

1. Stark T, Walker B, Phillips JK, Fejer R, Beck R. Handheld dynamometry correlation with the gold standard isokinetic dynamometry: A systematic review. PM R 2011;3(5):472-479; doi: 10.1016/j.pmrj.2010.10.025.

2. Martins JC, Teixeira-Salmela LF, Castro E Souza LA, Aguiar LT, Lara EM, Moura JB, et al. Reliability and validity of the modified sphygmomanometer test for the assessment of strength of upper limb muscles after stroke. J Rehabil Med. 2015;47(8):697-705; doi: 10.2340/16501977-1978.

3. Harris JE, Eng JJ. Strength training improves upperlimb function in individuals with stroke: a meta-analysis. Stroke. 2010;41(1):136-140; doi: 10.1161/STROKEAHA.109.567438.

4. Miller JE, Higgins LD, Dong Y, Collins JE, Bean JF, Seitz AL, et al. Association of Strength Measurement with Rotator Cuff Tear in Patients with Shoulder Pain: The Rotator Cuff Outcomes Workgroup Study. Am J Phys Med Rehabil. 2016;95(1):47-56, doi: 10.1097/PHM.0000 000000000329.

5. Saccol MF, Zanca GG, Ejnisman B, de Mello MT, Mattiello SM. Shoulder rotator strength and torque steadiness in athletes with anterior shoulder instability or SLAP lesion. J Sci Med Sport. 2014;17(5):463-468; doi: 10.1016/j.jsams.2013.10.246.
6. Burkhart SS, Morgan CD, Kibler WB. The disabled throwing shoulder: Spectrum of pathology Part I: Pathoanatomy and biomechanics. Arthroscopy. 2003;19(4): 404-420; doi: 10.1053/jars.2003.50128.

7. Cools AM, Palmans T, Johansson FR. Age-related, sportspecific adaptions of the shoulder girdle in elite adolescent tennis players. J Athl Train. 2014;49(5):647653; doi: 10.4085/1062-6050-49.3.02.

8. Harput G, Guney H, Toprak U, Kaya T, Filiz Colakoglu F, Baltaci G. Shoulder-Rotator Strength, Range of Motion, and Acromiohumeral Distance in Asymptomatic Adolescent Volleyball Attackers. J Athl Train. 2016;51(9): 733-738; doi: 10.4085/1062-6050-51.12.04.

9. Jung D-E, Moon D-C. Effect of the application of local vibration in scaption on joint stability. J Phys Ther Sci. 2015;27(1):115-116; doi.org/10.1589/jpts.27.115.

10. Paine R, Voight ML. The role of the scapula. Int J Sports Phys Ther. 2013 [cited 2017 Jan 19];8(5):617-629.

11. Conable KM, Rosner AL. A narrative review of manual muscle testing and implications for muscle testing research. J Chiropr Med. 2011;10(3):157-165; doi: 10.1016/ j.jcm.2011.04.001.

12. Bohannon RW. Manual muscle testing: does it meet the standards of an adequate screening test? Clin Rehabil.2005;19(6):662-667;doi:10.1191/0269215505cr873oa.

13. Katoh M, Hiiragi Y, Uchida M. Validity of Isometric Muscle Strength Measurements of the Lower Limbs Using a Hand-held Dynamometer and Belt: a Comparison with an Isokinetic Dynamometer. J Phys Ther Sci. 2011;23(4):553-557; doi: 10.1589/jpts.23.553.

14. Kim WK, Kim DK, Seo KM, Kang SH. Reliability and validity of isometric knee extensor strength test with hand-held dynamometer depending on its fixation: A pilot study. Ann Rehabil Med. 2014;38(1):84-93; doi: 10.5535/arm.2014.38.1.84.

15. Lu T-W, Chien H-L, Chang L-Y, Hsu H-C. Enhancing the examiner's resisting force improves the validity of manual muscle strength measurements: application to knee extensors and flexors. J Strength Cond Res. 2012;26(9):2364-2371; doi: 10.1519/JSC.0b013e31823 db080.

16. Bohannon RW, Bubela DJ, Wang Y-C, Magasi SR, Gershon RC. Adequacy of Belt-Stabilized Testing of Knee Extension Strength. J Strength Cond Res. 2011;25(7): 1963-1967; doi: 10.1519/JSC.0b013e3181e4f5ce.

17. Silva SM, Corrêa FI, Silva PFC, Silva DFT, Lucareli PRG, Corrêa JCF. Validation and reliability of a modified sphygmomanometer for the assessment of handgrip strength in Parkinson's disease. Braz J Phys Ther. 2015; 19(2):137-145; doi: 10.1590/bjpt-rbf.2014.0081.

18. Toohey LA, De Noronha M, Taylor C, Thomas J. Is a sphygmomanometer a valid and reliable tool to measure the isometric strength of hip muscles? A systematic review. Physiother Theory Pract. 2015;31(2):114-119; doi: 10.3109/09593985.2014.963905.

19. Martins JC, Aguiar LT, Lara EM, Teixeira-Salmela LF, Faria CDC de M. Assessment of grip strength with the 


\section{HUMAN MOVEMENT}

A.C. Barbosa et al., Validity reliability sphygmomanometer shoulder

modified sphygmomanometer test: Association between upper limb global strength and motor function. Braz J Phys Ther. 2015;19(6):498-506; doi: 10.1590/bjptrbf.2014.0118.

20. Souza LAC, Martins JC, Moura JB, Teixeira-Salmela LF, De Paula FVR, Faria CD. Assessment of muscular strength with the modified sphygmomanometer test: what is the best method and source of outcome values? Braz J Phys Ther. 2014;18(2):191-200; doi: 10.1590/S141335552012005000149.

21. Perossa DR, Dziak M, Vernon HT, Hayashita K. The intra-examiner reliability of manual muscle testing of the hip and shoulder with a modified sphygmomanometer: a preliminary study of normal subjects. J Can Chiropr Assoc. 1998;42(2):73-82.

22. Tyler TF, Nahow RC, Nicholas SJ, McHugh MP. Quantifying shoulder rotation weakness in patients with shoulder impingement. J Shoulder Elbow Surg. 2005;14(6): 570-574, 10.1016/j.jse.2005.03.003.

23. Aguiar LT, Lara EM, Martins JC, Teixeira-Salmela LF, Quintino LF, Christo PP, et al. Modified sphygmomanometer test for the assessment of strength of the trunk, upper and lower limbs muscles in subjects with subacute stroke: reliability and validity. Eur J Phys Rehabil Med. 2016;52(2):637-649; [cited 2017 Jan 18].

24. Lu Y-M, Lin J-H, Hsiao S-F, Liu M-F, Chen S-M, Lue Y-J. The Relative and Absolute Reliability of Leg Muscle Strength Testing by a Handheld Dynamometer. J Strength Cond Res. 2011;25(4):1065-1071, doi: 10.1519/JSC. 0b013e3181d650a6.

25. Schrama PPM, Stenneberg MS, Lucas C, Van Trijffel E. Intraexaminer reliability of hand-held dynamometry in the upper extremity: A systematic review. Arch Phys Med Rehabil. 2014;95(12):2444-2469; doi: 10.1016/j. apmr.2014.05.019.

26. Martin HJ, Yule V, Syddall HE, Dennison EM, Cooper C, Aihie Sayer A. Is hand-held dynamometry useful for the measurement of quadriceps strength in older people? A comparison with the gold standard biodex dynamometry. Gerontology. 2006;52(3):154-159; doi: 10.1159/000091824.

27. Deones VL, Wiley SC, Worrell T. Assessment of Quadriceps Muscle Performance by a Hand-Held Dynamometer and an Isokinetic Dynamometer. J Orthop Sport Phys Ther. 1994;20(6):296-301, doi: 10.2519/jospt.1994. 20.6.296.

28. Scott DA, Bond EQ, Sisto SA, Nadler SF. The intra- and interrater reliability of hip muscle strength assessments using a handheld versus a portable dynamometer anchoring station. Arch Phys Med Rehabil. 2004;85(4): 598-603; doi: 10.1016/j.apmr.2003.07.013.

29. Kollock RO, Onate JA, Van Lunen B. The reliability of portable fixed dynamometry during hip and knee strength assessments. J Athl Train. 2010;45(4):349-356; doi: 10.4085/1062-6050-45.4.349.

30. Barbosa AWC, Guedes CA, Bonifácio DN, de Fátima Silva A, Martins FLM, Almeida Barbosa MCS. The Pilates breathing technique increases the electromyographic amplitude level of the deep abdominal muscles in untrained people. J Bodyw Mov Ther. 2015;19(1):57-61, doi: 10.1016/j.jbmt.2014.05.011.

31. Moseley Jr. JB, Jobe FW, Pink M, Perry J, Tibone J. EMG analysis of the scapular muscles during a shoulder rehabilitation program. Am J Sports Med. 1992;20(2): 128-134; doi: 10.1177/036354659202000206.

32. Soares A. Correlation between handgrip, scapular and lumbar dynamometry tests [Correlação entre os testes de Dinamometria de Preensão Manual, Escapular e Lombar, in Portuguese]. Rev Acta Bras do Mov Hum. 2012;2(1):65-72.

33. Portney LG, Watkins MP. Foundations of Clinical Research: Applications to Practice. Survey of Ophthalmology. 2009;47(6); 598. doi: 10.1016/S0039-6257(02)00362-4. 